\title{
Lesiones neoplásicas sincrónicas en el cáncer colorrectal. Análisis de posibles factores que favorezcan su presentación
}

\author{
A. Borda, J. M. Martínez-Peñuela ${ }^{1}$, M. Muñoz-Navas, C. Prieto² ${ }^{2}$ M. Betés y F. Borda ${ }^{2}$ \\ Departamento de Digestivo. Clínica Universitaria de Navarra. ${ }^{I}$ Servicios de Anatomía Patológica y de ${ }^{2}$ Digestivo. \\ Hospital de Navarra. Pamplona
}

\section{RESUMEN}

Objetivo: en el cáncer colorrectal son poco conocidas las causas del frecuente desarrollo de lesiones neoplásicas sincrónicas. Pretendemos identificar posibles factores que pudieran influir en la multicentricidad lesional. Su conocimiento sería útil para, tras el tratamiento de las lesiones iniciales, optimizar el seguimiento en los pacientes que los presentaran.

Pacientes y métodos: estudiamos retrospectivamente 382 cánceres colorrectales diagnosticados mediante colonoscopia completa y estudio de la pieza quirúrgica. Comparamos una serie de parámetros referentes a los antecedentes personales y familiares, hábitos, datos clínicos y del tumor entre los grupos con y sin lesiones neoplásicas sincrónicas, mediante análisis estadístico univariable y multivariable.

Resultados: doscientos ocho $(54,5 \%)$ pacientes presentaron adenomas sincrónicos y 28 (7,3\%) carcinoma sincrónico. En el análisis multivariable el sexo masculino: $\mathrm{OR}=1,97$; $\mathrm{IC}=1,13-3,45$, $p=0,017$; la edad superior a 59 años: $\mathrm{OR}=2,57 ; \mathrm{IC}=1,54-4,29$, $\mathrm{p}<0,001$; el antecedente personal de pólipo colónico: $\mathrm{OR}=3,04$, $\mathrm{IC}=1,04-8,85, \mathrm{p}=0,042$ y el carácter obstructivo del cáncer: $\mathrm{OR}=0,48 ; \mathrm{IC}=0,27-0,85, \mathrm{p}=0,012$ se asocian significativamente con la multicentricidad lesional.

Conclusión: en los enfermos con cáncer colorrectal, nuestro estudio muestra una serie de parámetros, de fácil determinación, que podrían comportarse como factores de riesgo para el desarrollo de multicentricidad lesional. Estos factores deberán confirmarse mediante un estudio de seguimiento, analizando su comportamiento entre los pacientes que presenten $\mathrm{O}$ no lesiones metacrónicas tras la limpieza quirúrgico-endoscópica inicial.

Palabras clave: Cáncer colorrectal. Lesiones sincrónicas. Factores de riesgo.

\begin{abstract}
Aim: few data have been published regarding the causes of synchronous lesions in patients with colorectal cancer. The aim of our study was to identify potential factors that might be implicated in the development of multicentric lesions, since this knowledge could be useful for tailored follow-up once initial synchronous lesions have been removed.

Methods: we retrospectively reviewed 382 colorectal cancer cases diagnosed by total colonoscopy and histological study of surgical specimens. We divided our population into 2 groups, based on whether they had synchronous lesions or otherwise. Several data related to personal and family history, habits, symptoms, and tumor characteristics were assessed. Univariate and multivariate statistical analyses were performed.

Results: 208 (54.5\%) patients had synchronous adenomas and 28 (7.3\%) had synchronous cancer. A multivariate analysis showed that the following parameters were consistently related to the presence of multicentric lesions -male gender: $\mathrm{OR}=1.97$; $\mathrm{CI}=1.13-3.45 ; \mathrm{p}=0.017 ;$ age $\geq 59$ years: $\mathrm{OR}=2.57 ;$ $\mathrm{CI}=1.54-4.29 ; \mathrm{p}<0.001 ;$ personal history of colonic adenomas: $\mathrm{OR}=3.04 ; \mathrm{CI}=1.04-8.85 ; \mathrm{p}=0.042$; and obstructive tumors: $\mathrm{OR}=0.48 ; \mathrm{CI}=0.27-0.85 ; \mathrm{p}=0.012$.

Conclusion: our results show that several parameters that are easy to measure could be considered risk factors for the development of multicentric lesions. These factors need to be confirmed with follow-up studies analyzing their role in patients with and without metachronic lesions once all synchronous lesions have been removed.
\end{abstract}

Key words: Colorectal cancer. Synchronous lesions. Risk factors.

Borda A, Martínez-Peñuela JM, Muñoz-Navas M, Prieto C, Betés M, Borda F. Lesiones neoplásicas sincrónicas en el cáncer colorrectal. Análisis de posibles factores que favorezcan su presentación. Rev Esp Enferm Dig 2008; 100: 139-145.

Recibido: 27-08-07.

Aceptado: 08-01-08.

Correspondencia: Ana Borda. C/ Olite, 37, $7^{\circ}$ Izq. 31004 Pamplona. email: aborda@unav.es

\section{INTRODUCCIÓN}

El cáncer colorrectal constituye en la actualidad el tercero de los tumores malignos más frecuentes en la humanidad (1), siendo en España el segundo cáncer en cuanto 
a número de muertes ocasionadas (2). En nuestro país las estimaciones son de 25.000 nuevos diagnósticos y 12.500 fallecimientos/año (2). La gran mayoría de los cánceres colorrectales se desarrollan sobre un adenoma previo, siguiendo una secuencia multi-pasos adenoma-carcinoma (1-13) y siendo habitual la presencia de lesiones neoplásicas sincrónicas, adenomas o menos frecuentemente carcinomas, en el momento del diagnóstico (14-17). Se ha postulado que la multicentricidad tumoral pueda ser debida a la interacción de múltiples factores, entre los que cabría incluir determinada predisposición, tanto personal como familiar, mecanismos ambientales y/o relacionados con las características del tumor, aumentando así la probabilidad de desarrollar neoplasias en diferentes puntos del colon $(18,19)$.

Actualmente contamos con diversas publicaciones sobre el riesgo de presentar lesiones sincrónicas en pacientes con adenomas colorrectales $(15,16,20-23)$, pero tan sólo hemos podido encontrar un trabajo referente a la identificación de posibles factores de riesgo para el desarrollo de lesiones neoplásicas sincrónicas en el cáncer colorrectal (14). El hallazgo de diferencias entre series de cánceres colorrectales con y sin lesiones sincrónicas podría determinar una serie de posibles factores de riesgo para la multicentricidad tumoral. El control evolutivo del cáncer colorrectal está actualmente en discusión (3,2431). El conocimiento de posibles factores de multicentricidad podría facilitar un seguimiento más racional en cuanto a la detección de lesiones metacrónicas, tras la limpieza endoscópica-quirúrgica inicial. Finalmente, se facilitaría la posibilidad teórica de seleccionar subgrupos de pacientes que pudieran beneficiarse en un futuro de tratamientos con quimioprofilaxis, para intentar evitar la recidiva de las lesiones (32-35).

\section{MATERIAL Y MÉTODOS}

La serie incluye cánceres colorrectales diagnosticados durante el periodo 1999-2003 en la Clínica Universitaria de Navarra y en el Hospital de Navarra, mediante colonoscopia completa pre-operatoria o completada dentro de los 3 meses siguientes a la cirugía. El tumor debía haber sido resecado y confirmado histológicamente. Todos los estudios anatomopatológicos han sido revisados por un único patólogo, con el fin de unificar criterios y evitar diferencias inter-observador. Se han excluido los cánceres colorrectales que se presentasen en el seno de una poliposis múltiple familiar, o en pacientes con enfermedad inflamatoria intestinal.

Consideramos lesiones neoplásicas sincrónicas a la presencia de otro carcinoma colorrectal en el momento del diagnóstico o la de pólipos adenomatosos, tubulares y/o vellosos y adenomas serrados, sin incluir a los pólipos hiperplásicos.

Se ha etiquetado como cáncer sincrónico a la lesión que presentaba un estadio tumoral más bajo. También in- cluimos como cáncer sincrónico a los pólipos malignizados, con algún área de carcinoma en su interior.

Dividimos nuestra serie en dos grupos, según presentaran o no lesiones neoplásicas sincrónicas en el momento del diagnóstico de cáncer colorrectal. Estudiamos las posibles diferencias entre ambos grupos, con respecto a los siguientes parámetros: a) edad: analizándola en valores continuos y entre pacientes mayores y menores de 60 años; b) sexo; c) comunidad de residencia habitual: navarros o de cualquier otra comunidad; d) índice de masa corporal (IMC) dividiendo a los pacientes según la clasificación de la OMS (36) en peso inferior a la normalidad: IMC $<21$; normopeso: 21-24,9; sobrepeso: 25-29,9; y obesos: $>30$. Comparamos la frecuencia de casos con y sin lesiones sincrónicas para los puntos de corte del IMC de: $<21,<25$ y $\geq 30$; e) tabaco: como otros autores (37-39), consideramos fumador al que consumía 7 o más cigarrillos semanales, al menos durante 1 año antes del diagnóstico y ex-fumador al paciente que llevaba al menos 1 año sin fumar. Se analizan ambos grupos en cuanto a su condición de fumador y con respecto al número de cigarrillos consumidos, estableciendo los puntos de corte en $\geq 20, \geq 15, \geq 10 \mathrm{y} \geq 5$ cigarrillos/día; f) alcohol: no bebedores, bebedores de menos de 40 gramos y de más de 40 gramos de etanol/día, estudiando los pacientes de modo global y por sexos; g) antecedentes personales de: cáncer colorrectal, de cualquier tipo de cáncer previo y de pólipos adenomatosos colónicos; h) antecedentes de cáncer de cualquier tipo en familiares de primer grado. Todos estos antecedentes se han considerado de forma dicotómica: sí/no, según los datos de la historia clínica y/o encuesta enviada por correo; i) criterios revisados de Bethesda (40), considerando positivos los casos que cumplieran al menos 1 de sus 5 criterios; j) síntoma clínico predominante, basándonos en el motivo de solicitud de la colonoscopia diagnóstica, con la siguiente clasificación: i) antecedentes personales o familiares de pólipos o cáncer colorrectal; ii) chequeo de salud en paciente asintomático; iii) rectorragia; iv) dolor abdominal; v) alteración del ritmo intestinal, estreñimiento o diarrea; vi) anemia; vii) otros; k) limpieza en la colonoscopia diagnóstica: i) buena: correcta visualización de la mucosa; ii) regular: contenido líquido aspirable que permite un adecuado examen de la mucosa; y iii) mala: el contenido del colon es sólido o líquido no aspirable, que dificulta o impide el adecuado examen de la mucosa; 1) localización del cáncer colorrectal: recto, sigma, descendente, transverso y ascendente-ciego; m) carácter obstructivo del cáncer colorrectal: el que impide el paso del endoscopio, haciendo que la colonoscopia sea incompleta; n) tipo histológico del cáncer: para facilitar el estudio estadístico, se han agrupado los cánceres en adenocarcinomas y resto de tumores; o) grado de diferenciación del tumor (41): bien diferenciado, moderadamente diferenciado e indiferenciado; y p) estadificación del tumor según la clasificación pTNM del American Joint Comittee on Cancer ( $5^{\text {a }}$ edición) (42). Para el estudio estadístico se han agrupado en el mismo grupo los estadios 0 y I. 


\section{Análisis estadístico}

Los resultados se expresan como media aritmética y desviación típica, cuando las variables siguieron una distribución normal según el test de Kolmogorov-Smirnoff, y como mediana y rango en el resto de los casos. Las variables categóricas se expresan en frecuencias y porcentajes.

Las variables cuantitativas que siguieron una distribución normal se compararon mediante el test de $t$ de Student, en caso contrario se utilizó el test de U de Mann-Whitney. Las variables cualitativas se compararon mediante los test de Chi cuadrado y Fisher, determinando la odds ratio (OR), y el intervalo de confianza (IC) al 95\%. Se consideraron estadísticamente significativos los valores de $\mathrm{p}<0,05$.

\section{Analisis multivariante}

Se realizó mediante análisis de regresión logística múltiple, siendo la variable dependiente la presencia o no de lesiones sincrónicas y las independientes aquellas que en el estudio univariable mostraron una $p<0,20$. Se ha comprobado la existencia de interacciones, introduciendo el producto de las variables en el modelo.

\section{RESULTADOS}

Analizamos 382 pacientes con cáncer colorrectal, el 65,2 varones y el $34,8 \%$ mujeres. La mediana de edad fue de 65 años, rango $=20-88$. En 28 pacientes $(7,3 \%$ del global), se registró la presencia de cáncer sincrónico, todos ellos con adenomas sincrónicos. Diagnosticamos al menos un adenoma sincrónico en 208 pacientes (54,5\% del total). No se apreciaron diferencias significativas en cuanto al grado de limpieza del colon en la endoscopia diagnóstica entre los grupos sin y con lesiones neoplásicas sincrónicas: preparación buena $=68,6 \mathrm{vs} .70,5 \%$, regular $=17,3 \mathrm{vs}$. $22,3 \%$ y mala $=14,1$ vs. $7,25 \%(\mathrm{p}=0,46)$.
La edad del grupo con lesiones sincrónicas (mediana $=$ 67 años, rango $=30-83$ ) fue significativamente superior a la de los pacientes sin pólipos (mediana $=60$, rango $=20$ 88 años; $p<0,001)$. En la tabla I se representan las diferencias entre los cánceres colorrectales con y sin lesiones neoplásicas sincrónicas, con respecto a la edad de los pacientes expresada en menores y mayores de 60 años, sexo, comunidad de residencia habitual, antecedentes personales de pólipos de colon, de cáncer colorrectal, de cualquier otro cáncer, antecedentes de cáncer en familiares de primer grado y criterios de Bethesda revisados. Como vemos se confirma la mayor frecuencia de adenomas sincrónicos en el grupo de $\geq 60$ años: 64 vs. 37\%, $\mathrm{OR}=3,02$; IC95\%: 1,91-4,78; $\mathrm{p}<0,000001$. Las lesiones sincrónicas fueron significativamente más frecuentes en los varones: $62,7 \%$ con respecto a las mujeres, $39,1 \%$; $\mathrm{OR}=2,61 ; \mathrm{IC}=1,66-4,12 ; \mathrm{p}<0,0001$. Los cánceres con antecedentes personales de pólipos de colon mostraron una mayor tendencia a presentar lesiones sincrónicas: 68 vs. 48,8\%, sin llegar a alcanzar significación estadística $(\mathrm{p}=0,10)$.

La relación entre consumo de tabaco, toma de alcohol e índice de masa corporal con la frecuencia de lesiones sincrónicas se detalla en la tabla II. Los hábitos tóxicos han incidido fundamentalmente en el sexo masculino, siendo varones el $80,8 \%$ de los fumadores, el $88,5 \%$ de los bebedores y el $84,4 \%$ de los fumadores y bebedores. Registramos una mayor proporción de lesiones sincrónicas entre los casos que fuman y/o han fumado, que muestra tendencia a la significación estadística: fumadores $v s$. no fumadores: $\mathrm{p}=0,07$; fumadores-exfumadores $v s$. no fumadores: $p=0,098$. Los pacientes con lesiones sincrónicas mostraron un consumo más frecuente de 10 o más cigarrillos/día: 29/207 (14\%) vs. 14/175 (8\%), p = 0,064. Los otros puntos de corte estudiados mostraron diferencias menores entre ambos grupos: $\geq 20$ cigarrillos/día: $p$ $=0,15 ; \geq 15: \mathrm{p}=0,17 \mathrm{y} \geq 5$ cigarrillos/día: $\mathrm{p}=0,094$.

El $47 \%$ de los pacientes tomaba algo de alcohol, pero tan sólo un $4 \%$ de los enfermos consumía más de 40 g de

Tabla I. Factores personales y familiares y frecuencia de lesión sincrónica

\begin{tabular}{|c|c|c|c|c|c|}
\hline & \multicolumn{2}{|c|}{$\begin{array}{l}\text { No lesión } \\
\text { sincrónica }\end{array}$} & \multicolumn{2}{|c|}{$\begin{array}{c}\text { Lesión } \\
\text { sincrónica }\end{array}$} & \multirow[t]{2}{*}{$p$} \\
\hline & $n$ & $\%$ & $n$ & $\%$ & \\
\hline \multicolumn{6}{|l|}{ Edad } \\
\hline$\geq 60$ años & $89 / 247$ & 36 & $158 / 247$ & 34 & $<0,000001$ \\
\hline Resto & $85 / 135$ & 65,4 & $50 / 135$ & 34,6 & \\
\hline \multicolumn{6}{|l|}{ Sexo } \\
\hline Masculino & $93 / 249$ & 37,3 & $156 / 249$ & 62,7 & $<0,001$ \\
\hline Femenino & $81 / 133$ & 60,9 & $52 / 133$ & 39,1 & \\
\hline \multicolumn{6}{|l|}{ Comunidad de residencia } \\
\hline Navarra & $92 / 174$ & 52,9 & $109 / 208$ & 52,4 & 0,93 \\
\hline Resto & $82 / 174$ & 47,1 & $99 / 208$ & 47,6 & \\
\hline Antecendentes personales (pólipos de colon) & $144 / 295$ & 48,8 & $17 / 25$ & 68 & 0,10 \\
\hline Antecedentes personales (CCR) & $4 / 173$ & 2,3 & $7 / 208$ & 3,4 & 0,46 \\
\hline Antecedentes personales (otro cáncer) & $10 / 173$ & 5,8 & $17 / 207$ & 8,2 & 0,57 \\
\hline Antecedentes cáncer en familiares $1^{\text {er }}$ grado & $82 / 146$ & 56,2 & $83 / 170$ & 48,8 & 0,53 \\
\hline Criterios Bethesda revisados (al menos 1 criterio) & $64 / 131$ & 48,9 & $86 / 195$ & 44,1 & 0,40 \\
\hline
\end{tabular}


Tabla II. Tabaco, alcohol, masa corporal, sintomatología y frecuencia de lesiones

\begin{tabular}{|c|c|c|c|c|c|}
\hline & \multicolumn{2}{|c|}{$\begin{array}{l}\text { No lesión } \\
\text { sincrónica }\end{array}$} & \multicolumn{2}{|c|}{$\begin{array}{l}\text { Lesión } \\
\text { sincrónica }\end{array}$} & \multirow[t]{2}{*}{$p$} \\
\hline & $n$ & $\%$ & $n$ & $\%$ & \\
\hline \multicolumn{6}{|l|}{ Tabaco } \\
\hline No fumador & $91 / 164$ & 55,5 & $91 / 197$ & 46,2 & 0,14 \\
\hline Exfumador & $47 / 174$ & 28,7 & $61 / 197$ & 31 & \\
\hline Fumador & $26 / 164$ & 15,8 & $45 / 197$ & 22,8 & \\
\hline \multicolumn{6}{|l|}{ Alcohol (global) } \\
\hline No bebedor & $89 / 145$ & 61,4 & $81 / 176$ & 46 & 0,006 \\
\hline Bebedor & $56 / 145$ & 38,6 & $95 / 176$ & 54 & \\
\hline \multicolumn{6}{|l|}{ Alcohol (hombres) } \\
\hline No bebedor & $36 / 81$ & 44,4 & $41 / 131$ & 31,3 & 0,058 \\
\hline Bebedor & $45 / 81$ & 55,6 & $90 / 131$ & 68,7 & \\
\hline \multicolumn{6}{|l|}{ Alcohol (mujeres) } \\
\hline No bebedora & $55 / 69$ & 79,7 & $41 / 48$ & 85,4 & 0,43 \\
\hline Bebedora & $14 / 69$ & 20,3 & $7 / 48$ & 14,6 & \\
\hline \multicolumn{6}{|l|}{ Indice masa corporal } \\
\hline$<21$ & 9/174 & 5,2 & $3 / 208$ & 1,4 & 0,036 \\
\hline$<25$ & $52 / 174$ & 29,9 & $47 / 208$ & 22,6 & 0,094 \\
\hline$\geq 30$ & 29/129 & 22,5 & 29/143 & 20,3 & 0,66 \\
\hline \multicolumn{6}{|l|}{ Síntoma predominante } \\
\hline Rectorragia & $92 / 173$ & 53,2 & $113 / 207$ & 54,6 & 0,99 \\
\hline Anemia & $27 / 173$ & 15,6 & $29 / 207$ & 14 & \\
\hline Alteración ritmo intestinal & $20 / 173$ & 11,6 & $24 / 207$ & 11,6 & \\
\hline Dolor abdominal & $16 / 173$ & 9,2 & $18 / 207$ & 6,7 & \\
\hline Chequeo & $8 / 173$ & 4,6 & $10 / 207$ & 4,8 & \\
\hline Antecedente lesión colon & $5 / 173$ & 2,9 & $8 / 207$ & 3,9 & \\
\hline Otros & $5 / 173$ & 2,9 & $5 / 207$ & 2,4 & \\
\hline
\end{tabular}

etanol/día, por lo que el estudio estadístico se limita a comparar bebedores y no bebedores, sin establecer puntos de corte en el consumo de alcohol. Observamos una significativa mayor frecuencia de bebedores en la serie con lesiones sincrónicas: $\mathrm{p}=0,006 ; \mathrm{OR}=1,86$; IC $95 \%=$ $(1,16-2,99)$. Dividiendo por sexos se reducen las diferencias en los varones: $\mathrm{p}=0,058$ y se pierden en las mujeres: $\mathrm{p}=0,43$.

Tabla III. Localización, tipo histológico, grado de diferenciación del cáncer y frecuencia de lesiones sincrónicas

\begin{tabular}{|c|c|c|c|c|c|}
\hline & \multicolumn{2}{|c|}{$\begin{array}{l}\text { No lesión } \\
\text { sincrónica }\end{array}$} & \multicolumn{2}{|c|}{$\begin{array}{l}\text { Lesión } \\
\text { sincrónica }\end{array}$} & \multirow[t]{2}{*}{$p$} \\
\hline & $n$ & $\%$ & $n$ & $\%$ & \\
\hline Localización & & & & & 0,21 \\
\hline Recto & 79 & 48,2 & 77 & 37 & \\
\hline Sigma & 35 & 21,3 & 62 & 29,8 & \\
\hline Descendente & 8 & 4,9 & 12 & 5,8 & \\
\hline Transverso & 13 & 7,9 & 17 & 8,2 & \\
\hline Ascendente-ciego & 39 & 23,7 & 40 & 19,2 & \\
\hline Tipo histológico & & & & & 0,31 \\
\hline Adenocarcinoma & 151 & 87,8 & 173 & 83,6 & \\
\hline Resto de tipos & 21 & 12,2 & 34 & 16,4 & \\
\hline Grado diferenciación & & & & & 0,44 \\
\hline Bien diferenciado & 26 & 26,8 & 37 & 19,3 & \\
\hline Moderadamente & 102 & 65,8 & 132 & 68,7 & \\
\hline Indiferenciado & 27 & 17,4 & 23 & 12 & \\
\hline
\end{tabular}

El 3,1\% de los pacientes tuvieron un IMC $<21$, de ellos el 58,3\% fueron mujeres. El grupo con lesiones sincrónicas registró una frecuencia menor de pacientes con IMC < 21: 1,4 frente al 5,2\%, p = 0,036, OR =0,27, IC95\% $=0,06-1,10$, sin diferencias significativas entre los puntos de corte del IMC $\geq 30 \mathrm{y}<25$.

La síntomatología clínica que motivó la colonoscopia diagnóstica fue muy similar entre las series con y sin lesiones sincrónicas $(\mathrm{p}=0,99)$ (Tabla II).

En cuanto al estudio de los factores dependientes del cáncer observamos que, en los casos en que el carcinoma fue obstructivo, la frecuencia de adenomas fue del 34/88 $(38,6 \%)$, significativamente inferior a la de los cánceres no estenosantes: 173/293 (59\%); $\mathrm{p}<0,001$; OR = 0,44; $\mathrm{IC}=0,26-0,73$.

No hemos registrado diferencias significativas $(\mathrm{p}=$ $0,21)$ en cuanto al segmento anatómico del colon donde asentaba el cáncer y la presencia o no de lesiones sincrónicas, ni tampoco con respecto al tipo histológico del tumor y su grado de diferenciación (Tabla III).

La estadificación del tumor (pTNM) fue similar entre los grupos con y sin lesiones sincrónicas $(\mathrm{p}=0,79)$ (Fig. 1).

\section{Análisis estadístico multivariante}

En la tabla IV se recogen los resultados del modelo de análisis de regresión logística multivariable. La edad su- 


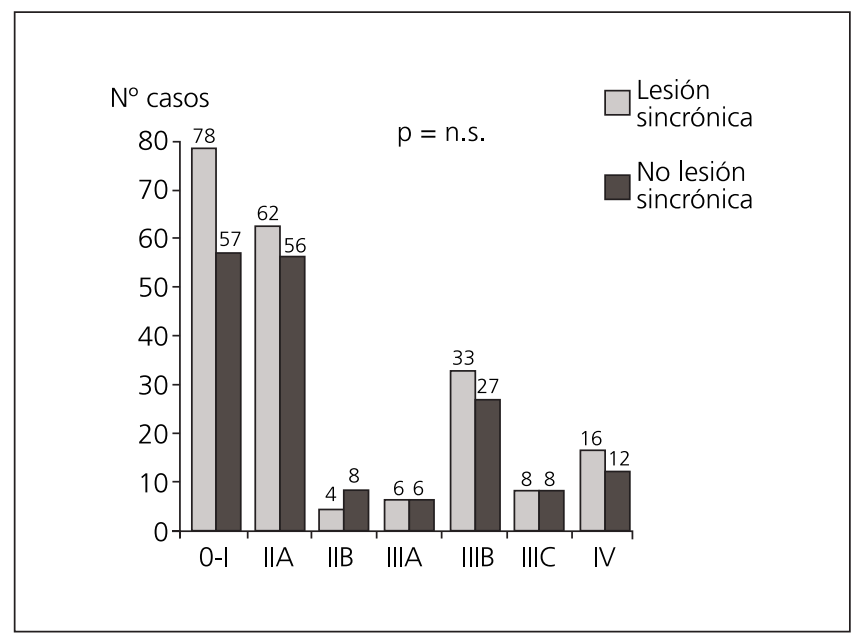

Fig. 1. Estadio del CCR y lesiones sincrónicas.

Tabla IV. Variables del análisis multivariante

\begin{tabular}{lccc}
\hline Variable & $p$ & Odds ratio & IC95\% \\
\hline Sexo masculino & 0,017 & 1,97 & $(1,13-3,45)$ \\
Edad $\leq 60$ años & $<0,01$ & 2,57 & $(1,54-4,29)$ \\
Alcohol & 0,196 & 1,42 & $(0,84-2,41)$ \\
Antecedente pólipos & 0,042 & 3,04 & $(1,04-8,85)$ \\
Carácter estenosante CCR & 0,012 & 0,48 & $(0,27-0,85)$ \\
\hline
\end{tabular}

perior a 59 años, el sexo masculino y los antecedentes personales de pólipos colónicos se relacionan con una mayor frecuencia de lesiones sincrónicas asociadas y el carácter obstructivo del cáncer se asocia a un menor riesgo para presentar adenomas asociados. El IMC y el consumo de tabaco no muestran diferencias significativas entre los grupos con y sin lesiones sincrónicas. El alcohol, a pesar de no estar asociado significativamente a las lesiones sincrónicas $(\mathrm{p}=0,19)$, ajusta el efecto del sexo sobre la frecuencia de dichas lesiones.

\section{DISCUSIÓN}

Ya se ha comentado la escasez de referencias bibliográficas acerca de los factores que pueden producir multicentricidad lesional en pacientes con cáncer colorrectal (14). La identificación de factores fácilmente accesibles (clínicos, personales o familiares, o de determinadas características del tumor que pudieran influir en la multiplicidad de las lesiones) sería importante para poder determinar un subgrupo de pacientes que precisasen un seguimiento más estricto tras la resección del tumor y la limpieza endoscópica de los pólipos (14). Hipotéticamente, este subgrupo de pacientes con mayor tendencia a la multicentricidad lesional podría incluso constituir una población diana para el empleo de terapias con quimioprofilaxis, como el ácido acetilsalicílico o inhibidores de la COX-2, actualmente en estudio (32-35), para evitar o reducir el riesgo de seguir presentando lesiones neoplásicas colónicas. Nuestro estudio pretende encontrar posibles factores de riesgo para el desarrollo de lesiones sincrónicas analizando la frecuencia de dichos posibles factores entre dos grupos de cáncer colorrectal, con y sin lesiones neoplásicas sincrónicas.

$\mathrm{Al}$ tratarse de un trabajo retrospectivo, nos enfrentamos a la limitación que supone la falta de algunos datos, insuficientemente detallados en las historias clínicas y fundamentalmente referidos a los antecedentes familiares y personales de los enfermos y sus hábitos tóxicos, que hemos intentado subsanar mediante el envío por correo de un cuestionario. No obstante, al incidir esta limitación en ambos grupos, con y sin lesiones sincrónicas, estimamos que no ha debido de ocasionar un sesgo importante a la hora de valorar nuestros resultados.

$\mathrm{Al}$ igual que en la única referencia bibliográfica encontrada (14), en los pacientes con cáncer colorrectal, su pertenencia al sexo masculino y el antecedente personal de adenomas colónicos se relacionan con una significativa mayor frecuencia de lesiones neoplásicas sincrónicas, lo que parece indicar un factor de riesgo para su presentación. Estos factores ya se habían postulado como favorecedores de multicentricidad en pacientes con adenomas de colon, que no habían desarrollado cáncer $(15,16,20,43)$. Por el contrario, en nuestro estudio no se confirma la posible influencia de otros parámetros como el antecedente familiar de cáncer gástrico, o de determinadas características del tumor como su localización proximal, el tipo histológico mucoide o el estadio T II, que en la serie de Piñol se describen como relacionadas con la multicentricidad lesional (14).

Como era esperable, la edad más avanzada se acompaña de una mayor frecuencia de lesiones sincrónicas, dato que ya se había descrito en series de pacientes con adenomas $(15,16)$.

Se ha publicado que tanto el hábito tabáquico como el alcohólico aumentan el riesgo de desarrollar lesiones neoplásicas colónicas (44-52), incluso a edades más precoces (44) y estando en discusión el posible factor del alcohol y el tabaco sobre el sistema de inestabilidad de microsatélites (53-57). Igualmente, se ha observado que los pacientes fumadores tienen un mayor riesgo de presentar nuevos adenomas tras la polipectomía endoscópica (58). En el análisis univariable, los consumos de tabaco y alcohol eran más frecuentes en los pacientes con lesiones sincrónicas, pero ambos hábitos estaban asociados con el sexo masculino. En el análisis multivariable el tabaco no se asoció significativamente con las lesiones sincrónicas, ni tampoco el consumo de alcohol. Al introducir el alcohol en el modelo se ajusta el efecto del sexo sobre la frecuencia de lesiones sincrónicas, confirmándose que el alcohol es un factor de confusión y el sexo masculino un factor de riesgo para el desarrollo de lesiones sincrónicas.

Se ha postulado que un aumento del IMC puede favorecer el desarrollo de adenomas y carcinomas colorrectales (59-63). En el análisis univariante la delgadez, expre- 
sada como IMC $<21$, parecía ejercer un factor protector sobre la presencia de lesiones sincrónicas. Al efectuar el estudio multivariante no se aprecian diferencias significativas, posiblemente debido al bajo número de pacientes con peso inferior a la normalidad y a su asociación con el sexo femenino.

Finalmente, nuestros casos de cáncer colorrectal con patrón obstructivo mostraron una menor frecuencia de lesiones sincrónicas. No podemos dar una explicación razonada a este hallazgo, ya que en la búsqueda bibliográfica efectuada la frecuencia de lesiones sincrónicas en este tipo de neoplasias oscila entre el 36 y el $58 \%(64,65)$, no siendo inferior con respecto al resto de los cánceres (64).

En resumen, y dentro de los enfermos con cáncer colorrectal, nuestro estudio ha mostrado una serie de parámetros muy fácilmente obtenibles: sexo masculino, edad más avanzada, antecedente personal de pólipos colónicos y carácter no obstructivo del tumor, que podrían comportarse como factores de riesgo para el desarrollo de multicentricidad lesional, delimitando un subgrupo de pacientes que precisasen un seguimiento postquirúrgico más estricto. Estos posibles factores de riesgo deberán confirmarse mediante un estudio de seguimiento tras la limpieza quirúrgico-endoscópica de las lesiones iniciales, analizando su comportamiento entre los pacientes que presenten o no lesiones metacrónicas. Este trabajo está siendo efectuado en la actualidad.

\section{BIBLIOGRAFÍA}

1. Weitz J, Koch M, Debus J, Höhler T, Galle PR, Büchler MW. Colorectal cancer. Lancet 2005; 365: 153-65.

2. Rodrigo L, Riestra S. Dieta y cáncer de colon. Rev Esp Enferm Dig 2007; 99: 183-9.

3. Castells A, Marzo M, Bellas B, Amador F, Lanas A, Mascortt J, et al. Guía de práctica clínica en prevención del cáncer colorrectal. Gastroenterol Hepatol 2004; 27: 573-634.

4. Atkin WS, Saunders BP. Surveillance guidelines after removal colorectal adenomatous polyps. Gut 2002; 51: V6-9.

5. Lynch JP, Hoops TC. The genetic pathogenesis of colorectal cancer. Hematol Oncol Clin N Am 2002; 16: 775-810.

6. Kondo Y, Iss JPJ. Epigenetic changes in colorectal cancer. Cancer Met Rev 2004; 23: 29-39.

7. Winawer SJ, Zauber AG. The advanced adenoma as the primary target of screening. Gastrointest Endosc Clin N Am 2002; 12: 1-9.

8. Yoo TW, Park DI, Kim YH, Kim WH, Kim TI, Kim HJ, et al. Clinical significance of small colorectal adenoma less than $10 \mathrm{~mm}$ : The KASID study. Hepatogastroenterol 2007; 54: 418-21.

9. Hermsen M, Postma C, Baak J, Rapallo A, Sciutto A, Roemen G, et al. Colorectal adenoma to carcinoma progression follows multiple pathways of chromosomal instability. Gastroenterol 2002; 123: 1109-19.

10. Jass JR. Colorectal cancer: A multipathway disease. Crit Rev Oncol 2006; 12: 273-87.

11. Cabrera CM, López-Nevot MA. APC and chromosome instability in colorectal cancer. Rev Esp Enferm Dig 2005; 97: 738-43.

12. Cruz-Bustillo Clarens D. Genomic instability and colorectal cancer. Rev Esp Enferm Dig 2005; 97: 683-7.

13. Caro-Patón Gómez A. Interleukins and colon cancer. Rev Esp Enferm Dig 2005; 97: 613-8.

14. Piñol V, Andreu M, Castells A, Paya A, Bessa X, Jover R. Synchronous colorectal neoplasms in patients with colorectal cancer: Predisposing individual and familial factors. Dis Col Rectum 2004; 47: $1192-2000$
15. Anderson JC, Alpern Z, Messina CR, Lane B, Hubbard P, Grimson R, et al. Predictors of proximal neoplasia in patients without distal adenomatous pathology. Am J Gastroenterol 2004; 99: 472-7.

16. Imperiale TF, Wagner DR, Lin CY, Larkin GN, Rogge JD, Ransohoff DF. Risk of advanced proximal neoplasms in asymptomatic adults according to the distal colorectal findings. N Engl J Med 2000; 343: 169-174.

17. Slater G, Fleshner P, Aufses AH Jr. Colorectal cancer location and synchronous adenomas. Am J Gastroenterol 1988; 83: 832-6.

18. Jass J, Withehall V, Yuong J, Legget B. Emerging concepts in colorectal neoplasia. Gastroenterol 2002; 123: 862-76.

19. Cannon-Albright LA, Skolnick MH, Bishop DT, Lee RG, Burt RW . Common inheritance susceptibility to colonic adenomatous polyps and associated colorectal cancer. N Engl J Med 1988; 319: 533-7.

20. Senore C, Signan N, Bonelli L, Sciallero S, Pennazio M, Angioli D, et al. Predicting proximal advanced neoplasms at screening sigmoidoscopy. Dis Colon Rectum 2004; 47: 1331-40.

21. Martínez ME, Sampliner R, Marshall JR, Bhattacharyya AK, Reid $\mathrm{ME}$, Alberts DS. Adenoma characteristics as risk factors for recurrence of advanced adenomas. Gastroenterol 2001; 120: 1077-83.

22. Lan YT, Lin JK, Li AF, Lin TC, Chen WS, Jiang JK et al. Metachronous colorectal cancer: Necessity of post-operative colonoscopic surveillance. Int J Colorectal Dis 2005; 20: 121-5.

23. Bonithon-Kopp C, Fenger PF, Cabeza E, O'Morain C, Kronborg O, Faivre J. Colorectal adenoma characteristis as predictors of recurrente. Dis Colon Rectum 2004; 47: 323-33.

24. Rodríguez-Moranta F, Salo J, Arcusa A, Boadas J, Piñol V, Bessa X, et al. Postoperative surveillance in patients with colorectal cancer who have undergone curative resection: A prospective, multicenter, randomized, controlled trial. J Clin Oncol 2006; 24: 386-93.

25. Desch CE, Benson AB, Somerfield MR, Flynn PJ, Krause C, Loprinzi CL, et al. Colorectal cancer surveillance: 2005 update of an American Society of Clinical Oncology practice guideline. J Clin Oncol 2005; 23: 8512-9.

26. Rex DK, Kahi CJ, Levin B, Smith RA, Bond JH, Brooks D, et al. Guidelines for colonoscopy surveillance after cancer resection: A consensus update by the American Cancer Society and the US MultiSociety Task Force on Colorectal Cancer. Gastroenterol 2006; 130: 1865-71.

27. Figueredo A, Rumble RB, Maroun J, Earle CC, Cummings B, McLeod R, et al. Follow-up of patients with curatively resected colorectal cancer: A practice guideline. BMC Cancer 2003; 3: 26.

28. Cubiella J, Gómez R, Sánchez E, Díez MS, Vega M. Seguimiento endoscópico tras resección curativa del cáncer colorrectal: resultados de un protocolo asistencial. Rev Esp Enferm Dig 2003; 95: 273-7.

29. Papagrigoriadis S. Follow-up of patients with colorectal cancer: The evidence is in favour but we are still in need of a protocol. Int J Surg 2007; 5: 120-8.

30. Jeffery M, Hickey BE, Hider PN. Follow-up strategies for patients treated for non-metastatic colorectal cancer Cochrane Database Syst Rev 2007; 1: CD002200.

31. Betés M, Carretero C, Muñoz-Navas M, editores. Evidencia científica en cribado del cáncer colorrectal. Manual de actuación. Madrid: IM\&B; 2006.

32. Dubé C, Rostov A, Lewin G, Tsertsvadze A, Barrowman N, Code C, et al. The use of aspirin for primary prevention of colorectal cancer: A systematic review prepared for the US Preventive Services Task Force. Ann Int Med 2007; 146: 365-75.

33. Asano TK, McLeod RS. Non steroidal anti-inflammatory drugs and aspirin for the prevention of colorectal adenomas and cancer: A systematic review. Dis Colon Rectum 2004; 47 (5): 665-73.

34. Bertagnolli MM. Chemoprevention of colorectal cancer with cyclooxygenase-2 inhibitors: Two steps forward, one step back. Lancet Oncol 2007; 8: 439-43.

35. Chan AT, Ogino S, Fuchs CS. Aspirin and the risk of colorectal cancer in relation to the expression of COX-2. N Engl J Med 2007; 356: 2131-42.

36. Word Health Organization. Physical status: The use of interpretation of anthropometry: Report of a WHO expert comittee. Word Health Organ Rech Rep Ser 1995; 854: 1160-67.

37. Cottone M, Rosselli M, Orlando A, Puleo A, Cappello M, Traina M. et al. Smoking habits and recurrence in Crohn's disease. Gastroenterology 1994; 106: 643-8. 
38. Godtfredsen NS, Prescott E, Osler M. Effect of smoking reduction on lung cancer risk. JAMA 2005; 294: 1505-10.

39. Cosnes J, Carbonnel F, Carrat F, Beaugerie L, Cattan S, Gendre J. Effects of current and former cigarette smoking on the clinical course of Crohn's disease. Aliment Pharmacol Ther 1999; 13: 1403-11.

40. Umar A, Boland CR, Terdiman JP, Syngal S, De la Chapelle A, Rüschoff J, et al. Revised Bethesda guidelines for hereditary nonpolyposis colorectal cancer (Lynch syndrome) and microsatellite instability. J Ntl Cancer Inst 2004; 96: 261-8.

41. Dukes CE, Bussey HJR. The spread of rectal cancer and its effect on prognosis. Br J Cancer 1958; 12: 309-20.

42. Greene FL, Page DL, Fleming ID, editors. AJCC (American Joint Committee on Cancer). Cancer Staging Manual. $6^{\text {th }}$ ed. New York: Springer-Verlag; 2002. p. 113.

43. Van Stolk RW, Beck GJ, Baron JA, Haile R, Summers R. Polyp Prevention Study Group. Adenoma characteristics at first colonoscopy as predictors of adenoma recurrence and characteristics at follow-up. Gastroenterol 1998; 115: 13-8.

44. Zisman AL, Nickolov A, Brand RL, Gorchow A, Roy HR. Associations between the age at diagnosis and location of colorectal cancer and the use of alcohol and tobacco. Arch Intern Med 2006; 166: 62934.

45. Chao A, Thun MJ, Jacobs EJ, Henley SJ, Rodríguez C, Calle EE. Cigarrette smoking and colorectal cancer mortality in the Cancer Prevention Study II. J Natl Cancer Inst 2000; 92: 1888-96.

46. Cho E, Smith-Warner SA, Ritz J, Van den Brandt PA, Colditz GA, Folsom AR, et al. Alcohol intake and rectal cancer: A pooled analysis of 8 cohort studies. Ann Intern Med 2004; 140: 603-13.

47. Giovannucci E, Martínez ME. Tobacco, colorectal cancer, and adenomas: A review of the evidence. J Natl Cancer Inst 1996; 88: 1717-30.

48. Giovannucci E. An updated review of the epidemiological evidence that cigarette smoking increases risk of colorectal cancer. Cancer Epidemiol Biomarkers Prev 2001; 10: 725-31.

49. Mizoue T, Inoue M, Tanaka K, Tsuji I, Wakai K, Nagata C, et al. Tobacco smoking and colorectal cancer risk: An evaluation based on a systematic review of epidemiological evidence among the Japanese population. Jpn J Clin Oncol 2006; 36: 25-39.

50. Mizoue T, Tanaka K, Tsuji T, Wakai K, Nagata C, Otani T, et al. Alcohol drinking and colorectal cancer risk: An evaluation based on a systematic review of epidemiological evidence among the Japanese population. Jpn J Clin Oncol 2006; 36: 582-97.

51. Ferry MB, Neugut AI. Cigarette smoking and the colorectal adenoma-carcinoma sequence: A hypothesis to explain the paradox. Am J Epidemiol 1998; 147: 903-10.

52. Slattery ML, Curtin K, Anderson K, Ma KN, Ballard L, Edwards S, et al. Associations between cigarette smoking, lifestyle factors, and mi- crosatellite instability in colon tumors. J Natl Cancer Inst 2000; 92: 1831-6.

53. Watson P, Ashwathnarayan R, Lynch HT, Roy HK. Tobacco use and increased colorectal cancer risk in patients with hereditary nonpolyposis colorectal cancer (Lynch Syndrome). Arch Intern Med 2004; 164: 2429-31.

54. Diergaarde B, Vrieling A, Van Kraats AA, Van Muijen GN, Kok FJ, Kampman E. Cigarette smoking and genetic alteration in sporadic colon carcinomas. Carcinogenesis 2003; 24: 565-71.

55. Solera Albero J, Tárraga López PJ, Carbayo Herencia JA, López Cara MA, Celada Rodríguez A, Cerdán Oliver M, et al. Influence of diet and lifestyle in colorectal cancer. Rev Esp Enferm Dig 2007; 99: 190200 .

56. Slattery ML, Anderson K, Curtin K, Ma KN, Schaffer D, Samowitz $\mathrm{W}$, et al. Dietary intake and microsatellite instability in colon tumours. Int J Cancer 2001; 93: 601-7.

57. Roy HK, Gulizia JM, Karolski WJ, Ratashak A, Sorrell MF, Tuma D. Etanol promotes intestinal tumorogenesis in the MIN mouse: Multiple intestinal neoplasia. Cancer Epidemiol Biomarkers Prev 2002;11: 1499-502.

58. Jacobson JS, Neugut AI, Murria T, Garbowski GC, Forde KA, Treta $\mathrm{MR}$, et al. Cigarette smoking and other behavioral risk factors to recurrente of colorectal adenomatous polyps (New York City, USA). Cancer Causes Control 1994; 5: 215-20.

59. Frezza EE, Wachtel MS, Chiriva-Internati M. Influence of obesity on the risk on developing colon cancer. Gut 2006; 55: 285-91.

60. Pischon T, Laman PH, Boeing H, Friedenreich C, Norat T, Tjonneland A, et al. Body size and risk of colon and rectal cancer in the European Prospective Investigation Into Cancer and Nutrition (EPIC). J Natl Cancer Inst 2006; 98: 920-31.

61. Tamakoshi K, Wakai K, Kojima M, Watanabe Y, Hayakawa N, Toyoshima $\mathrm{H}$, et al. A prospective study of body size and colon cancer mortality in Japan: The JACC Study. Int Obes Relat Metab Disord 2004; 28: 551-8.

62. Sedjo RL, Byers T, Levin TR, Haffner SM, Saad MF, Tooze JA, et al. Change in body size and the risk of colorectal adenomas. Cancer Epidemiol Biomarkers Prev 2007; 16: 526-31.

63. Jacobs ET, Martínez ME, Alberts DS, Jiang R, Lance P, Lowe KA, et al. Association between body size and colorectal adenoma recurrence. Clin Gastroenterol Hepatol 2007; 5: 982-90.

64. Bat L, Neumann G, Shemesh E. The association of synchronous neoplams with occluding colorectal cancer. Dis Colon Rectum 1985; 28 : 149-51.

65. Vitale MA, Villotti G, d'Alba L, Frontespezi S, Iacopini F, Iacopini G. Preoperative colonoscopy after self-expandable metallic stent placement in patients with acute neoplastic colon obstruction. Gastrointest Endosc 2006; 63: 814-9. 